\title{
Current challenges in photosynthesis: from natural to artificial
}

\author{
Harvey J. M. Hou ${ }^{1 *}$, Suleyman I. Allakhverdiev ${ }^{2,3}$, Mohammad M. Najafpour $^{4}$ and Govindjee ${ }^{5}$ \\ ${ }^{1}$ Department of Physical Sciences, Alabama State University, Alabama, AL, USA \\ 2 Institute of Plant Physiology, Russian Academy of Sciences, Moscow, Russia \\ ${ }^{3}$ Institute of Basic Biological Problems, Russian Academy of Sciences, Moscow, Russia \\ ${ }^{4}$ Department of Chemistry, Center of Climate Change and Global Warming, Institute for Advanced Studies in Basic Sciences, Zanjan, Iran \\ ${ }^{5}$ Departments of Biochemistry and Plant Biology, Center of Biophysics and Quantitative Biology, University of Illinois at Urbana-Champaign, Urbana, IL, USA \\ ${ }^{*}$ Correspondence: hhou@alasu.edu
}

Edited and reviewed by:

Steven Carl Huber, USDA-ARS, USA

Keywords: photosynthesis, artificial photosynthesis, water oxidation, thylakoid, chlorophyll $f$

Photosynthesis is a process by which plants, algae, cyanobacteria, and anoxygenic photosynthetic bacteria capture and store solar energy on a massive scale, in particular via the water-splitting chemistry (Hoganson and Babcock, 1997; Blankenship, 2002; Ferreira et al., 2004; Loll et al., 2005; Yano et al., 2006; Umena et al., 2011). It is the most important reaction on Earth, estimated to produce more than 100 billion tons of dry biomass annually; this means that photosynthesis is producing biomass equal to two Egyptian pyramids per hour. But, this will not be enough to sustain life on Earth by the year 2050. The global fossil fuels on which we currently depend are derived from millions of years of past photosynthetic activity. The fossil energy fuels are limited and must be replaced by renewable and environment-friendly energy source to support and sustain life on Earth (Lewis and Nocera, 2006; Blankenship et al., 2011). To address this immediate energy crisis, worldwide efforts are being made on artificial photosynthesis using the principles and mechanisms observed in nature (Brimblecombe et al., 2009; McConnell et al., 2010; Kanady et al., 2011; Wiechen et al., 2012; Najafpour et al., 2013). It is not a matter of mimicking natural photosynthesis, but to use its current knowledge to improve photosynthesis itself, as well as to produce biofuels, including hydrogen evolution by artificial means (Barber, 2009; Hou, 2010; Nocera, 2012; He et al., 2013).

This book contains 10 chapters and presents recent advances in photosynthesis and artificial photosynthesis. It starts with two opinion articles on possible strategies to improve photosynthesis in plants and fascinating mechanisms of unidirectional photodamage of pheophytin in photosynthesis. The idea that plant photosynthesis is maximized due to the perfect evolution might be faulty. Leister evaluated and argued the issue openly and proposed that improvement of photosynthesis can be made by synthetic biology including genetic engineering, redesign or de novo creation of entire photosystems as well as conventional breeding (Leister, 2012). Unidirectional photodamage of a pheophytin molecule in photosystem II and purple bacterial reaction centers was observed. The mysterious phenomena were analyzed and discussed in terms of different possible functions of the pheophytin in photosynthesis (Hou, 2014).

The book is followed by four review articles that discuss the current state of research on: photosynthetic water oxidation in natural and artificial photosynthesis, as obtained by mass spectrometry (MS) and Fourier transform infrared spectroscopy (FTIR); functional models of thylakoid lumen; and horizontal gene transfer in photosynthetic eukaryotes. The time-resolved isotope-ratio membrane-inlet mass spectrometry (TR-IR-MIMS) is able to determine the isotopic composition of gaseous products. Shevela et al briefly introduced the key aspects of the methodology, summarized the recent results on the mechanisms and pathways of oxygen formation in PS II using this unique technique and outlined the future perspectives of the application in water splitting chemistry (Shevela and Messinger, 2013) Another unique technique in probing the mechanism of water oxidation in PS II is the light-induced FTIR difference spectroscopy. Chu reviewed the recent fruitful structural data, and believed that the FTIR will continue to provide vital structural and mechanistic insights into the water-splitting process in PS II together with isotopic labeling, site-directed mutagenesis, model compound studies, and computational calculation (Chu, 2013). The thylakoid lumen offers the environment for oxygen evolution, electron transfer, and photoprotection in photosynthesis. Jarvi et al evaluated the recent studies of many lumen proteins and highlighted the importance of the thiol-disulfide modulation in controlling the functions of the thylakoid lumen proteins and their pathways of photosynthesis (Järvi et al., 2013). Qiu et al discussed that importance of the horizontal gene transfer (HGT) in enriching the algal genomes and proposed that the alga endosymbionts may be the HGT vectors in photosynthetic eukaryotes (Qiu et al., 2013).

Finally, the book offers four research articles, which focus on FTIR studies on photosynthetic reaction centers, functions of thylakoid protein kinases STN7 and STN8, photosynthesis acclimation of maize seedlings, and characterization of the newly discovered chlorophyll $f$-containing cyanobacterium Halomicronema hongdechloris. The computational calculation (ONIOM) is increasingly critical in interpreting the FTIR data in elucidating the structural and functional relationship in photosynthesis. Zhao et al using ONIOM type calculation to simulate isotope edited FTIR difference spectra for reaction centers with a variety of foreign quinones in the $\mathrm{Q}_{\mathrm{A}}$ site and allows a direct assessment of the appropriateness of previous IR assignments and suggestions (Zhao et al., 2013). The protein kinases STN7 and STN8 are predominately responsible for the thylakoid 
phosphorylation in PS II. Wunder et al reported the effects of the STN8 expression levels on the formation and modulation of thylakoid proteins and kinases (Wunder et al., 2013). Hirth et al assessed the photosynthetic acclimation responses of the C3 and C4 plants under simulated field light conditions (Hirth et al., 2013). Recently a chlorophyll $f(\mathrm{Chl} f)$ in cyanobacterium Halomincronema hongdechloris was identified and has the most red-shifted absorption peak of $707 \mathrm{~nm}$ in oxygenic photosynthesis (Chen et al., 2010), which may enhance the potential photosynthesis efficiency for solar fuel production. The Halomincronema hongdechloris was characterized upon the exposure to the different light, $\mathrm{pH}$, salinity, temperature, and nutrition to achieve the optimizing growth culture conditions (Li et al., 2014).

Due to the extremely limited time frame for collecting manuscripts and the strict deadline for publishing this book, several planned manuscripts by world leaders, who had agreed to contribute, are unfortunately not included in this book. Thus, the current book provides a snapshot of the latest work in photosynthesis research. To obtain complete information on the current progress in the field of photosynthesis, we highly recommend reviews and research articles, published in 2013, in two volumes of Photosynthesis Research (Allakhverdiev et al., 2013a,b)

In conclusion, the book provides readers with some of the most recent and exciting breakthroughs from natural to artificial photosynthesis, discusses the potential limitations of the results, and addresses open questions in photosynthesis and energy research. It is written by 31 young active scientists and established leading experts from Australia, Finland, Germany, Sweden, Taiwan, and the United States. We hope that this book is able to provide novel and insightful information to readers and stimulate the future research endeavors in the photosynthesis community.

\section{ACKNOWLEDGMENTS}

We take this opportunity to acknowledge and thank all the authors for writing excellent book chapters, and for being supportive and cooperative when their manuscripts were being reviewed and revised. We also thank the external reviewers for their timely contribution and effort in judging the value of the manuscript and delivering constructive comments, which have undoubtedly improved the quality and readability of the book. We thank the Specialty Chief Editor of Frontiers in Plant Science, Steve Huber, for his valuable advice and insightful discussions. We also thank Kennedy Wekesa and Audrey Napier for critical reading of the manuscript. We are very grateful to Amanda Baker, Graeme Moffat, Despoina Evangelakou, and Adriana Timperi of the Frontiers Production Office for their insightful advice and meaningful assistance during the entire book project.

\section{REFERENCES}

Allakhverdiev, S. I., Shen, J.-R., and Edwards, G. E. (2013a). Special issues on photosynthesis education honoring govindjee. Photosynthesis Res. 116, 1-534.

Allakhverdiev, S. I., Shen, J.-R., and Edwards, G. E. (2013b). Special issues on photosynthesis education honoring govindjee. Photosynthesis Res. 117, 1-566.

Barber, J. (2009). Photosynthetic energy conversion: natural and artificial. Chem. Soc. Rev. 38, 185-196 doi: 10.1039/b802262n

Blankenship, R. E. (2002). Molecular Mechanisms of Photosynthesis. Oxford: Blackwell Science. doi: 10.1002/9780470758472
Blankenship, R. E., Tiede, D. M., Barber, J., Brudvig, G. W., Fleming, G., Ghirardi, M., et al. (2011). Comparing the efficiency of photosynthesis with photovoltaic devices and recognizing opportunities for improvement. Science 332, 805-809 doi: 10.1126/science. 1200165

Brimblecombe, R., Dismukes, G. C., Swiegers, G. F., and Spiccia, L. (2009). Molecular water-oxidation catalysts for photoelectrochemical cells. Dalton Trans. 43, 9374-9384 doi: 10.1039/b912669d

Chen, M., Schliep, M., Willows, R. D., Cai, Z. L., Neilan, B. A., and Scheer, H. (2010). A red-shifted chlorophyll. Science 329, 1318-1319. doi: 10.1126/science.1191127e.1

Chu, H.-A. (2013). Fourier transform infrared difference spectroscopy for studying the molecular mechanism of photosynthetic water oxidation. Front. Plant Sci. 4:146. doi: 10.3389/fpls.2013.00146

Ferreira, K. N., Iverson, T. M., Maghlaoui, K., Barber, J., and Iwata, S. (2004). Architecture of the photosynthetic oxygen-evolving center. Science 303, 1831-1838. doi: 10.1126/science.1093087

He, W., Zhao, K.-H., and Hou, H. J. M. (2013). Toward solar fuel production using manganese/semiconductor systems to mimic photosynthesis. NanoPhotoBioSciences 1, 63-78.

Hirth, M., Dietzel, L., Steiner, S., Ludwig, R., Weidenbach, H., Pfalz, J., et al. (2013). Photosynthetic acclimation responses of maize seedlings grown under artificial laboratory light gradients mimicking natural canopy conditions. Front. Plant Sci. 4:334. doi: 10.3389/fpls.2013.00334

Hoganson, C. W., and Babcock, G. T. (1997). A metallo radical mechanism for the generation of oxygen from water in photosynthesis. Science 277, 1953-1956. doi: $10.1126 /$ science.277.5334.1953

Hou, H. J. M. (2010). Structural and mechanistic aspects of Mn-oxo and Cobased compounds in water oxidation catalysis and potential application in solar fuel production. J. Integr. Plant Biol. 52, 704-711 doi: 10.1111/j.17447909.2010.00974.x

Hou, H. J. M. (2014). Unidirectional photodamage of pheophytin in photosynthesis. Front. Plant Sci. 4:554. doi: 10.3389/fpls.2013.00554

Järvi, S., Gollan, P. J., and Aro, E.-M. (2013). Understanding the roles of the thylakoid lumen in photosynthesis regulation. Front. Plant Sci. 4:434. doi: 10.3389/fpls.2013.00434

Kanady, J. S., Tsui, E. Y., Day, M. W., and Agapie, T. (2011). A synthetic model of the $\mathrm{Mn}_{3} \mathrm{Ca}$ subsite of the oxygen-evolving complex in photosystem II. Science 333, 733-736. doi: 10.1126/science. 1206036

Leister, D. (2012). How can the light reactions of photosynthesis be improved in plants? Front. Plant Sci. 3:199. doi: 10.3389/fpls.2012.00199

Lewis, N. S., and Nocera, D. G. (2006). Powering the planet: chemical challenges in solar energy utilization. Proc. Natl. Acad. Sci. U.S.A. 103, 15729-15735 doi: 10.1073/pnas.0603395103

Li, Y., Lin, Y., Loughlin, P. C., and Chen, M. (2014). Optimization and effects of different culture conditions on growth of Halomicronema hongdechloris - a filamentous cyanobacterium containing chlorophyll f. Front. Plant Sci. 5:67. doi: $10.3389 /$ fpls.2014.00067

Loll, B., Kern, J., Saenger, W., Zouni, A., and Biesiadka, J. (2005). Towards complete cofactor arrangement in the 3.0A resolution structure of photosystem II. Nature 438, 1040-1044. doi: 10.1038/nature04224

McConnell, I., Li, G., and Brudvig, G. W. (2010). Energy conversion in natural and artificial photosynthesis. Chem. Biol. 17, 434-447. doi: 10.1016/j.chembiol.2010.05.005

Najafpour, M. M., Mahnaz Abasi, M., and Allakhverdiev, S. I. (2013). Recent proposed mechanisms for biological water oxidation. NanoPhotoBioSciences 1, 79-92.

Nocera, D. G. (2012). The artificial leaf. Acc. Chem. Res. 45, 767-776. doi: $10.1021 / \operatorname{ar} 2003013$

Qiu, H., Yoon, H. S., and Bhattacharya, D. (2013). Alga lendosymbionts as vectors of horizontal gene transfer in photosynthetic eukaryotes. Front. Plant Sci. 4:366. doi: 10.3389/fpls.2013.00366

Shevela, D., and Messinger, J. (2013). Studying the oxidation of water to molecular oxygen in photosynthetic and artificial systems by time-resolved membraneinlet mass spectrometry. Front. Plant Sci. 4:473. doi: 10.3389/fpls.2013. 00473

Umena, Y., Kawakami, K., Shen, J. R., and Kamiya, N. (2011). Crystal structure of oxygen-evolving photosystem II at a resolution of 1.9 A. Nature 473, 55-61. doi: 10.1038 /nature09913 
Wiechen, M., Berends, H.-M., and Kurz, P. (2012). Water oxidation catalysed by manganese compounds: from complexes to "biomimetic rocks." Dalton Trans. 41, 21-31. doi: 10.1039/c1dt11537e

Wunder, T., Xu, W., Liu, Q., Wanner, G., Leister, D., and Pribil, M. (2013). The major thylakoid protein kinases STN7 and STN8 revisited: effects of altered STN8 levels and regulatory specificities of the STN kinases. Front. Plant Sci. 4:417. doi: $10.3389 /$ fpls.2013.00417

Yano, J., Kern, J., Sauer, K., Latimer, M. J., Pushkar, Y., Biesiadka, J., et al. (2006). Where water is oxidized to dioxygen: structure of the photosynthetic $\mathrm{Mn}_{4} \mathrm{Ca}$ cluster. Science 314, 821-825. doi: 10.1126/science. 1128186

Zhao, N., Lamichhane, H. P., and Hastings, G. (2013). Comparison of calculated and experimental isotope edited FTIR difference spectra for purple bacterial photosynthetic reaction centers with different quinones incorporated into the $\mathrm{Q}_{\mathrm{A}}$ binding site. Front. Plant Sci. 4:328. doi: 10.3389/fpls.2013. 00328
Conflict of Interest Statement: The authors declare that the research was conducted in the absence of any commercial or financial relationships that could be construed as a potential conflict of interest.

Received: 26 March 2014; accepted: 09 May 2014; published online: 28 May 2014. Citation: Hou HJM, Allakhverdiev SI, Najafpour MM and Govindjee (2014) Current challenges in photosynthesis: from natural to artificial. Front. Plant Sci. 5:232. doi: $10.3389 / \mathrm{fpls} .2014 .00232$

This article was submitted to Plant Physiology, a section of the journal Frontiers in Plant Science.

Copyright (c) 2014 Hou, Allakhverdiev, Najafpour and Govindjee. This is an openaccess article distributed under the terms of the Creative Commons Attribution License (CC BY). The use, distribution or reproduction in other forums is permitted, provided the original author(s) or licensor are credited and that the original publication in this journal is cited, in accordance with accepted academic practice. No use, distribution or reproduction is permitted which does not comply with these terms. 